\title{
Autofocusing drift tube linac envelopes
}

\author{
Olivier Shelbaya $\odot,{ }^{1,2, *}$ Tiffany Angus $\odot,{ }^{1}$ Rick Baartman, ${ }^{1,2}$ Paul M. Jung, ${ }^{1,2}$ Oliver Kester, ${ }^{1,2}$ \\ Spencer Kiy, ${ }^{1,2}$ Thomas Planche, ${ }^{1,2}$ and Stephanie D. Rädel ${ }^{1}$ \\ ${ }^{1}$ TRIUMF, 4004 Wesbrook Mall, Vancouver BC, V6T 2A3, Canada \\ ${ }^{2}$ Department of Physics and Astronomy, University of Victoria, Victoria BC, V8W 2Y2, Canada
}

(Received 19 October 2021; accepted 6 December 2021; published 21 December 2021)

\begin{abstract}
To date, beam dynamics studies and design of combined zero degree drift tube linac (DTL) structures (Kombinierte Null Grad Struktur; KONUS) have only been carried out in multiparticle codes. Quantities such as the beam envelopes are obtained by averaging over particles, whose tracking is computationally intensive for large bunch populations. Tune computations, which depend on this average, are burdensome to obtain. This has motivated the implementation of a simulation of KONUS DTLs in the code TRANSOPTR, whose Hamiltonian treatment of beam dynamics enables the integration of energy gain from the longitudinal electric field on axis while simultaneously elaborating the field in transverse directions to obtain the linear optics. The code also features an in-built space charge capability. The evolution of the beam matrix including longitudinal optics is computed in a reference Frenet-Serret frame through the timedependent DTL cavity fields. This enables fast envelope simulations for DTLs, resulting in a variable energy sequential tune optimization capability. The implementation methodology and optimization techniques, applicable for any combination of DTL tanks and bunchers, is outlined. Comparisons with the code LORASR, in addition to beam-based $E / A$ measurements of a DTL are presented.
\end{abstract}

DOI: $10.1103 /$ PhysRevAccelBeams.24.124602

\section{INTRODUCTION}

Tuning accelerators requires modeling of the beam's evolution through the machine, producing simulated diagnostic fiducials for on-line comparison. Such models are also used in parallel to machine operation, improving understanding of established operational tunes. This can help optimize tuning procedures, by providing operators a better understanding of the machine's expected response to optics adjustments. The complexity of recent linac designs has also increased [1], for example using dedicated rf devices such as bunchers, RFQ structures, or interdigital $\mathrm{H}$-mode $(\mathrm{IH})$ cavities. As a result, studying global machine tunes frequently requires use of several dedicated simulation codes to represent subsections of the accelerator. The configuration space of such devices can also be quite large. Unlike for example a dedicated driver linac, typically operated at constant intensity and output properties, variable energy, multi-cavity linac structures such as CERN's REX-ISOLDE [2], the GSI injectors HSI (Hochstrominjektor; High Current Injector) and HLI (Hochladungsinjektor; High Charge State Injector) [3] or

\footnotetext{
*oshelb@triumf.ca
}

Published by the American Physical Society under the terms of the Creative Commons Attribution 4.0 International license. Further distribution of this work must maintain attribution to the author(s) and the published article's title, journal citation, and DOI. most recently FRIB [4] present the challenge of accommodating beams of different energy, charge and mass through control of electric field scaling and phasing. The transverse tune must be separately defined using groups of quadrupoles or solenoids. Separated function DTLs like the ISAC-I linac [5] apply the KONUS [6] prescription using dedicated accelerating tanks and bunchers, each with independent $\mathrm{rf}$ [7] equipment. Unless a large number of bunch diagnostics are available throughout the accelerator, it can be exceedingly difficult and time-consuming to establish precise tunes. Having to model a system with this many parameters highlights the need for a fast optimizable code. To this end, the envelope code TRANSOPTR has been adapted to simulate and optimize multiparameter, variable energy envelopes for separated function DTL structures. The novel capability including KONUS sections is demonstrated for the first time, using the TRIUMF ISAC-DTL (Fig. 1) as an example.

Though there have been model-based studies of ISAC linac beam dynamics [8,9] using TRACE3D [10], TRACK [11], LANA [12], and LORASR [13,14], none of them have allowed full simulations of the end-to-end behavior of the accelerator. This has further motivated [15] the development of the implementation presented herein. The envelope code TRANSOPTR [16-18] itself has been in use at TRIUMF since 1984 [19]. Ongoing development has resulted in a capability to simulate acceleration in axially symmetric, time dependent electric fields such as the electron linear 


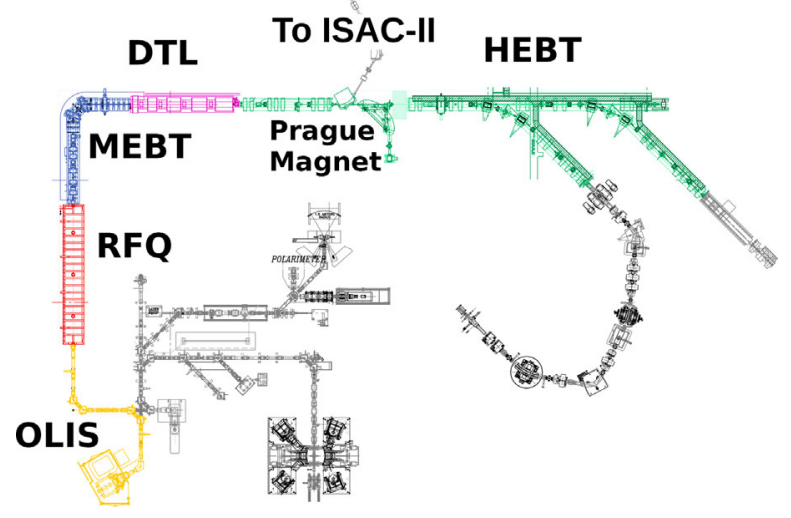

FIG. 1. Overview of the ISAC-I linear accelerator complex. The offLine ion source (OLIS) provides stable pilot beams. Beam energy is measured at the Prague (HEBT1) high energy analyzing magnet station, a $90^{\circ}$ dipole which provides a reading of energy and energy spread.

accelerator (e-linac) [20] or nonaxially symmetric cases such as a cyclotron rf gap. The code also features an RFQ linac capability [21]. Since TRANSOPTR is fast, being approximately equivalent to integrating the trajectories of only four particles, it is well suited for use during machine tuning.

We present in this work the novel drift tube linac (DTL) beam envelope capability in the code TRANSOPTR, in particular its ability to perform constrained optimization problems upon the beam or transfer matrices through a DTL structure. Cavity longitudinal electric fields, required by TRANSOPTR, have been generated using OPERA2D [22] and machine blueprints. Computed beam envelopes are compared to those from a LORASR [14] model of the ISACDTL. The latter is used as an example of a particle code which properly represents drift tube acceleration. The infinitesimal transfer matrix approach means energy gain from a time varying electric field can be directly integrated in TRANSOPTR without resorting to stored "transit time factors." This method is general; while the ISAC-DTL is shown in this paper, other linacs can be implemented with ease, requiring only the on axis field distributions. A comparison with beambased energy measurements is also presented, showcasing TRANSOPTR's usefulness for tune computations and analysis of a DTL structure. Though not applicable for present ISAC cases, it is noted that TRANSOPTR is capable of a native firstorder space charge implementation for all simulated elements (see Refs. [17,21,23,24]), now including drift tube linacs.

\section{TRANSPORT, ENVELOPES}

Matrix methods applied to linear optics are well known and in the 1950s came to be applied to the developing field of particle beam optics. Penner [25] extended these transverse dynamics to include $\delta$, the particle's fractional forward momentum deviation compared with the momentum of the reference particle, and thereby could include dispersion. In the 1960s, work at SLAC by Karl Brown,
Charles Moore, Hal Butler, Sam Howry and Barbara Kear and then Christoph Iselin (CERN), Frank Rothacker (SLAC), and David Carey (Fermilab) [26], developed a computer code, TRANSPORT [27], that could transport the coordinates through a system by multiplying together the matrices of the individual elements. This technique was restricted to elements whose matrices could be found analytically and so could not describe real elements unless they had been idealized as having piece-wise constant strengths. For example, quadrupoles, dipoles, and solenoids were restricted to hard-edged with fields constant along the reference trajectory. Even for linear order, this made the calculations more or less approximate. In some cases, the approximation could be improved by combining elements' matrices with edge-effect matrices that had been derived by integrating the equations of motion and applying them as kicks. This "fringe field integral" approach was developed especially by Wollnik and co-workers [28], which otherwise had capabilities and restrictions similar to those of TRANSPORT.

The formalism they developed and that became a standard, included all six phase space dimensions in a state vector $\mathbf{X}:=\left(x, x^{\prime}, y, y^{\prime}, l, \delta\right)$, where $x^{\prime}=P_{x} / P, y^{\prime}=P_{y} / P$, and $\delta=\Delta P / P$. The fifth coordinate was $l$, the path length difference compared with the reference on-momentum particle. This was sufficient for describing transport systems composed of idealized magnetic elements in a static and nonaccelerating system, where $P$ is a constant. TRANSOPTR extended the capability, releasing these constraints, by generalizing those coordinates in a simple way, as will be shown.

\section{A. The sigma matrix and envelope equations}

The key advance that made the original TRANSPORT so useful was the extension of the matrix formalism of single particle optics to cover that of beams. This was accomplished by introducing the "beam matrix": a general ellipsoid can be represented by a matrix, the beam or "sigma" matrix, and its evolution when acted upon by linear forces can be derived from the same transfer matrix $\mathbf{M}$ as used for individual trajectories.

$$
\sigma_{\mathrm{f}}=\mathbf{M} \sigma_{\mathrm{i}} \mathbf{M}^{T} .
$$

The formalism was used in TRANSPORT and quickly seen $[29,30]$ to be generalizable by interpreting the beam matrix as simply the covariance matrix of the phase space coordinates of the ensemble of particles:

$$
\boldsymbol{\sigma}:=\frac{1}{N} \sum_{n=1}^{N} \mathbf{X} \mathbf{X}^{T} .
$$

The diagonal of this matrix was then seen no longer as the squares of the radii in each phase space coordinate 
boundary (the envelope), but reinterpreted as the meansquared size in that direction.

Separately (in a land far far away...), Kapchinsky and Vladimirsky [31] had, in 1959, augmented the known "envelope equation" for uncoupled linear transverse motion, to include space charge, but only for the specialized distributions (the "KV" distribution) that have exactly linear transverse space charge force. Sacherer (1970) then showed [29] that this envelope equation can be derived from the sigma matrix formalism. He showed that this envelope equation applies even in cases of nonideal $\mathrm{KV}$ distributions, if it is interpreted as the covariance matrix. He further extended the results to bunched beams of ellipsoidal form.

Naturally as space charge applies continuously, to describe the evolution of the covariance matrix requires to write it in differential form:

$$
\frac{\mathrm{d} \boldsymbol{\sigma}}{\mathrm{d} s}=\mathbf{F}(s) \boldsymbol{\sigma}+\boldsymbol{\sigma} \mathbf{F}(s)^{\mathrm{T}} .
$$

This is found from Eq. (1) by replacing $\mathbf{M}$ with $\mathbf{I}+\mathbf{F d} s$ where $\mathbf{F}$ is the "inifinitesimal transfer matrix" and $\mathbf{I}$ is the identity.

\section{TRANSOPTR}

Originally, TRANSOPTR was written to duplicate TRANSPORT's capabilities, but with expanded and more flexible fitting abilities [16]. The transport system is written as a sequence of calls in FORTRAN rather than as a sequence of coded cards, as is done in TRANSPORT. In 1983 [23], it was expanded to include space charge, but not in the usual technique of modeling space charge kicks as individual thin lens defocus kicks. Rather, a new mode was incorporated, to directly integrate the Sacherer ordinary differential equations above (3). This opened the possibility of modeling elements that do not have analytically integrable equations of motion, including for example any kind of element with soft edges: soft-edged quadrupoles, dipoles, and solenoids could be handled simply by providing the strength as function of the independent variable $s$, the distance along the reference trajectory. A technique used for speed in many legacy codes such as TRACE3D and TRANSPORT is to use stored transfer matrices for idealized elements. Here the gain in computation speed is minimal over integrating through all elements. TRANSOPTR solves the envelope equation (3) numerically, given initial conditions, using a Runge-Kutta (RK) method with an adaptive step size [32,33]. The method provides a self-consistent error estimate based on a symplecticity check, which allows the user to specify a single dimensionless tolerance parameter $\epsilon$.

\section{A. Time and the fifth coordinate}

TRANSPORT used as fifth coordinate the "path length difference" with respect to the reference particle. But then on specifying the fifth parameter of the bunch characteristics card, the users' manual [34] asks for "the longitudinal extent of the bunch of particles." These two, path length difference and bunch extent, do not correspond to each other unless the bunch is ultrarelativistic. For nonrelativistic particles, the bunch length in the lab frame is its time difference between head and tail, multiplied by its speed. As originally written, the resulting matrices to describe the dynamics included no dynamical quantities, only geometrical ones. A user had to correct the results for speed effects, and spread in speed resulting from momentum spread, in order to make sense of the results. For particles with a spread in speed, the TRANSPORT coordinate 5 corresponded to no simply measurable parameter. In TRANSOPTR, the fifth coordinate is simply arrival time difference with respect to the reference particle, multiplied by the speed of the reference particle. This makes it the longitudinal analog of the transverse coordinates $x$ and $y$. Moreover, multiplying this coordinate by $2 \pi /(\beta \lambda)$, one can obtain the relative phase of a particle in an $\mathrm{rf}$ device.

A legacy of the TRANSPORT longitudinal coordinate is that the word "isochronous" is often used incorrectly. Brown admitted: It is somewhat unfortunate that this word has been used in the literature [for cases where TRANSPORT finds $\left.\mathbf{M}_{56}=0\right]$, since it is applicable only to highly relativistic particles [27]. The proper terminology for this case is "isohodous," meaning path lengths are equal for different momenta. In the TRANSOPTR case, $\mathbf{M}_{56}=0$ does actually mean the transport is isochronous.

As the fifth coordinate is time, the sixth should be its natural canonical conjugate, energy. In particular, in the coordinate system that moves and orients with the reference trajectory (the Frenet-Serret system), it is the energy difference with respect to the reference particle's energy, divided by the reference particle's speed. To sum up: $\mathbf{X}=\left(x, P_{x}, y, P_{y},-\beta c \Delta t, \Delta E /(\beta c)\right)$. This has also the immediate advantage that electrostatic elements or any $\mathrm{rf}$ device described with the aid of a scalar potential can be handled naturally for small momentum offsets, since the canonical energy $E$ contains the potential energy component:

$$
c^{2} P \Delta P=(E-q \phi) \Delta E .
$$

Moreover, outside of devices with scalar electric potential, $\Delta E /(\beta c)=\Delta P$ and so when normalized by momentum, agrees with TRANSPORT for any magnetic element. In this way, TRANSOPTR can handle acceleration by a simple extension.

\section{B. Hamiltonian for the DTL}

As usual, we use the coordinate system that moves and orients with the reference trajectory (the Frenet-Serret system), and have the distance along the reference trajectory $s$ as the independent variable. Then the Hamiltonian is $-P_{s}$ : 


$$
H\left(x, P_{x}, y, P_{y}, t, E ; s\right)=-q A_{s}-\sqrt{\left(\frac{E-q \Phi}{c}\right)^{2}-m^{2} c^{2}-\left(P_{x}-q A_{x}\right)^{2}-\left(P_{y}-q A_{y}\right)^{2}}
$$

The first four particle coordinates are differentials as they are the transverse positions and momenta with respect to the reference particle. The fifth coordinate is time, the sixth should be energy. We need to transform these to $(\beta c \Delta t, \Delta E /(\beta c))$, as indicated above.

\section{Potentials}

As shown in a previous work [32,33], the vector potential, $\mathbf{A}$ to lowest order in the transverse coordinates for an axially symmetric $\mathrm{rf}$ gap is $A_{x}=A_{y}=0$ and

$$
A_{s}=-\mathcal{E}(s)\left(1-\frac{\omega^{2}}{c^{2}} \frac{x^{2}+y^{2}}{4}\right) \frac{\sin (\omega t+\theta)}{\omega},
$$

and the scalar potential is

$$
\Phi=\mathcal{E}^{\prime} \cos (\omega t+\theta) \frac{x^{2}+y^{2}}{4} .
$$

The function $\mathcal{E}(s)$ is the amplitude of the on axis electric field, $\omega$ and $\theta$ are its frequency and phase.

The energy and time equations for the reference particle are

$$
\begin{gathered}
\frac{\mathrm{d} E}{\mathrm{~d} s}=\frac{\partial H}{\partial t}=q \mathcal{E} \cos (\omega t+\theta), \\
\frac{\mathrm{d} t}{\mathrm{~d} s}=-\frac{\partial H}{\partial E}=\frac{E}{P c^{2}}=\frac{1}{\beta_{0} c} .
\end{gathered}
$$

(From here on, we drop the 0 subscript: $\beta$ and $\gamma$ are implicitly assumed to be the relativistic parameters of the reference particle).

These give the functions $E_{0}(s)$ and $t_{0}(s)$ about which $t$ and $E$ are expanded: $E=E_{0}+\Delta E$ and $t=t_{0}+\Delta t$. So, we transform the canonical variables $t$ and $-E$ to $(\Delta t,-\Delta E)$, using as generating function

$$
G=-\left(t-\int \frac{\mathrm{d} s}{\beta(s) c}\right)\left(\Delta E+E_{0}\right) .
$$

(Check: $\frac{\partial G}{\partial t}=-E, \frac{\partial G}{\partial(-\Delta E)}=\Delta t$.) The Hamiltonian gets the added terms

$$
\frac{\partial G}{\partial s}=\frac{\Delta E+E_{0}(s)}{\beta(s) c}-\Delta t E_{0}^{\prime}(s) .
$$

Finally, we transform from $(\Delta t,-\Delta E)$ to $\left(z, P_{z}\right):=$ $(-\beta c \Delta t, \Delta E /(\beta c))$. The reason for the sign change is as follows: an early arrival implies $\Delta t<0$, but this means the particle is ahead so $z>0$. The generating function is

$$
G=-\beta c \Delta t P_{z}
$$

(Check: $\frac{\partial G}{\partial \Delta t}=-\Delta E, \frac{\partial G}{\partial P_{z}}=z$.) The term to be added to the Hamiltonian is

$$
\frac{\partial G}{\partial s}=\frac{\beta^{\prime}}{\beta} z P_{z}=\frac{\gamma^{\prime}}{\beta^{2} \gamma^{3}} z P_{z}=\frac{q \mathcal{E} C}{\beta c P \gamma^{2}} z P_{z} .
$$

$\left[C:=\cos \left(\omega t_{0}(s)+\theta\right), S:=\sin \left(\omega t_{0}(s)+\theta\right).\right]$

Now, expanding the new Hamiltonian which is a function of $\mathbf{X}:=\left(x, P_{x}, y, P_{y}, z, P_{z}\right)$, we get the result

$$
\begin{aligned}
H(\mathbf{X} ; s)= & \frac{P_{x}^{2}}{2 P}+\frac{P_{y}^{2}}{2 P}+\frac{q}{2 \beta c}\left(\mathcal{E}^{\prime} C-\mathcal{E} S \frac{\omega \beta}{c}\right) \frac{r^{2}}{2} \\
& +\frac{P_{z}^{2}}{2 \gamma^{2} P}+\frac{q \mathcal{E} C}{\beta c} \frac{z P_{z}}{\gamma^{2} P}-\frac{q \mathcal{E} \omega S}{\beta^{2} c^{2}} \frac{z^{2}}{2} .
\end{aligned}
$$

We can recognize individual terms. (i) The factor in parentheses represents the focal power of an rf gap, e.g., a buncher and (ii) Taking the limit as $\omega \rightarrow 0$ reproduces the known Hamiltonian of the DC accelerator [20]: note that in that case, $\mathcal{E}^{\prime}=-\phi^{\prime \prime}$.

\section{Infinitesimal transfer matrix $\mathbf{F}$}

Now that the Hamiltonian for linear motion (Eq. (14) has been obtained, it is a simple matter to find the infinitesimal transfer matrix F. Writing the equations of motion $\left(x^{\prime}=\partial H / \partial P_{x}, \quad P_{x}^{\prime}=-\partial H / \partial x\right.$, etc. $)$, the following $\mathbf{F}$ matrix is found for the axially symmetric linear accelerator:

$$
\mathbf{F}=\left(\begin{array}{cccccc}
0 & \frac{1}{P} & 0 & 0 & 0 & 0 \\
\mathcal{A}(s) & 0 & 0 & 0 & 0 & 0 \\
0 & 0 & 0 & \frac{1}{P} & 0 & 0 \\
0 & 0 & \mathcal{A}(s) & 0 & 0 & 0 \\
0 & 0 & 0 & 0 & \frac{\beta^{\prime}}{\beta} & \frac{1}{\gamma^{2} P} \\
0 & 0 & 0 & 0 & \mathcal{B}(s) & -\frac{\beta^{\prime}}{\beta}
\end{array}\right),
$$

where we have defined

$$
\mathcal{A}(s)=\frac{-q}{2 \beta c}\left(\mathcal{E}^{\prime} C-\mathcal{E} S \frac{\omega \beta}{c}\right), \quad \mathcal{B}(s)=\frac{q \mathcal{E} \omega S}{\beta^{2} c^{2}} .
$$


This matrix has been coded as a subroutine into TRANSOPTR. Along with two additional Eqs. (8) and (10), this sets up to calculate through any axially symmetric linac or buncher. Function $\mathcal{E}(s)$ is input as normalized values on a set of points $\left(\mathcal{E}_{\mathrm{n}}\left(s_{i}\right)\right.$; usually 10 per cell is sufficient), which are then spline interpolated and scaled during calculation by a factor $V_{0}$, the field amplitude: $\mathcal{E}(s)=V_{0} \mathcal{E}_{\mathrm{n}}(s)$.

\section{TRANSOPTR MODEL OF THE ISAC-DTL}

The ISAC-DTL features five accelerating cavities and three bunching cavities, following an $8 \mathrm{~m}$ long, $35 \mathrm{MHz}$ RFQ, with separated prebunching [35] and injection boosting [36] capability. The RFQ accelerates beams to a constant $E / A=0.153 \mathrm{MeV} / u$ for $1 \leq A / q \leq 30$. After optional charge increase with a stripping foil [37], the $106 \mathrm{MHz}$ ISAC-DTL (Fig. 2) allows for fully variable output energy acceleration with $0.117 \mathrm{MeV} / u \leq E / A \leq 1.8 \mathrm{MeV} / u$ [9], with typical operation at $1.53 \mathrm{MeV} / u$. For any energy, the drift tube cavities are powered on and tuned sequentially, from Tank-1 onward. Each cavity is set to its operational $E / A$ (Table I). This procedure is followed until the desired energy is attained, with the last needed tank being driven at a lower field amplitude if necessary. The TRANSOPTR implementation, via Eq. (15), requires values for the on axis longitudinal electric field distribution, necessary to compute energy gain and the resulting rf optical effects of acceleration. Models representing the ISAC-DTL's IH cavities longitudinal electric fields were constructed in the Poisson solver OPERA2D $[22,38]$ and the simulated on axis electric fields are shown in Fig. 3, along with tube effective voltages for operational acceleration during beam delivery.

\section{A. Code implementation}

The DTL's lattice was implemented in TRANSOPTR referring to original blueprints and publications $[5,39,40]$ which were also used to position the cavity fields (Fig. 3) and quadrupole triplets [41]. Fields for the triple-gap

\section{DRIFT TUBE LINAC}

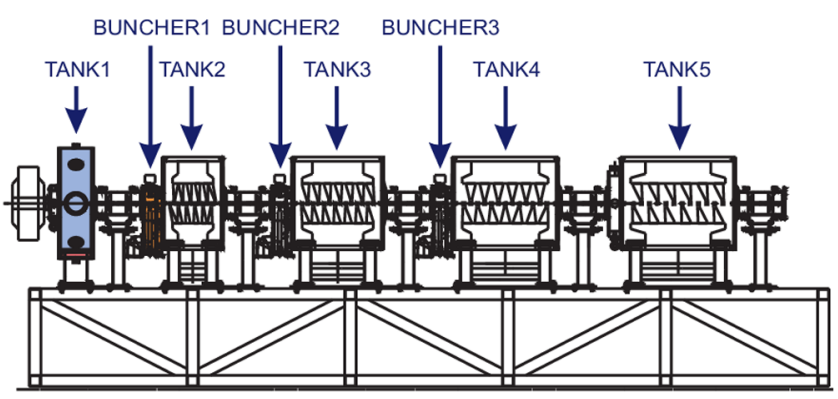

FIG. 2. The separated function ISAC-DTL. Quadrupole triplets along the lattice define the transverse optics. Courtesy of M. Marchetto, TRIUMF.
TABLE I. Typical operational energy configuration of the ISAC-DTL.

\begin{tabular}{lc}
\hline \hline Tank & $E / A(\mathrm{MeV} / \mathrm{u})$ \\
\hline RFQ output & 0.153 \\
IH Tank-1 & 0.238 \\
Buncher-1 & 0.254 \\
IH Tank-2 & 0.439 \\
Buncher-2 & 0.461 \\
IH Tank-3 & 0.781 \\
Buncher-3 & 0.803 \\
IH Tank-4 & 1.149 \\
IH Tank-5 & 1.530 \\
\hline \hline
\end{tabular}

bunchers can be found in Refs. [38,42]. TRANSOPTR also tracks the evolution of the particle's global time vs the independent variable $s$; the distance along the reference orbit. This allows for the more faithful simulation of transittime effects between rf cavities. In TRANSOPTR, beam transport or acceleration systems are coded directly at the subroutine level and compiled once prior to execution. It is thus straightforward to use all or parts of the accelerator simulation within loops or other algorithms to investigate the behavior of individual variables such as rf phases or amplitudes in a DTL accelerating tank. Figure 4 shows the characteristically nonsinusoidal and nonlinear energyphase relationship emerge along the length of Tank-3, at fixed electric field amplitude but varied rf phase. TRANSOPTR can be called to perform such rf cavity grid scans with ease through PYTHON, which also facilitates the postprocessing of output data, all within the same program. Since it is at its heart a design and optimization code, constraints can be imposed upon the beam matrix at any point inside the DTL, including inside the rf cavities. The optimizer, either downhill simplex or simulated annealing [19], varies user-specified parameters in search of a solution, while solving the envelope equation, along with the time and energy coordinates of the reference. An example is the setting of triplet quadrupole gradients, which is achieved by constraining the quadrupoles to produce mirrored transverse phase space distributions across the triplet, or transfer matrix elements $\mathbf{M}_{11}=-1$ and $\mathbf{M}_{12}=0$. Entire DTL tunes can thus be computed without referring to previously saved values for the transverse optics. Another considerable advantage to TRANSOPTR's reference particle and envelope approach is that such beam dynamics investigations are decoupled from transmission considerations. A separate PYTHON package has been developed at TRIUMF which automates the generation, execution, and manipulation of TRANSOPTR files, further enhancing its ease of use. A centralized database is maintained, containing coordinates and properties for each device in the accelerator. This way, arbitrary sequences of elements can be generated for analysis. The code is also in use as the physics engine for a series of 

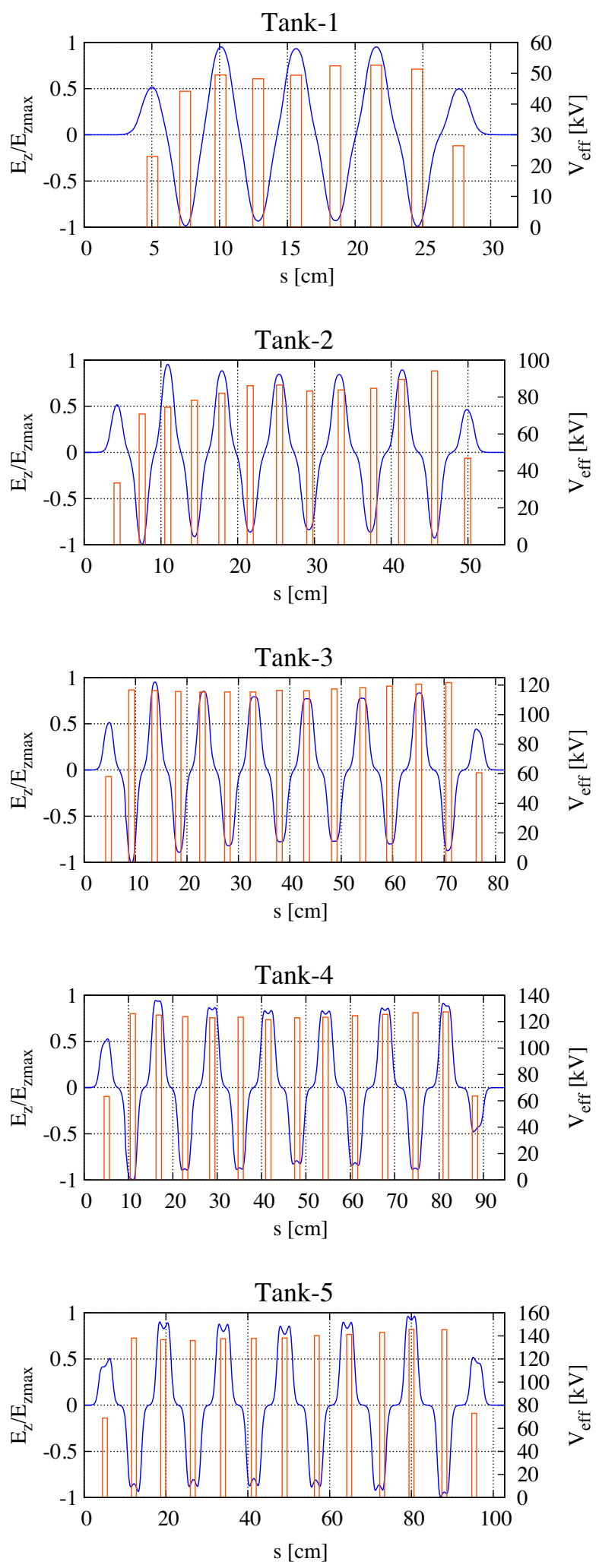

FIG. 3. OPERA2D computed normalized on axis electric fields $\left(E_{Z} / E_{\max }:=\mathcal{E}_{\mathrm{n}}(s)\right)$ for Tanks $1-5$ of the ISAC-DTL. Note the nonflatness of the peak electric field intensity across the cavities. Gap effective voltages for an ${ }^{14} \mathrm{~N}^{3+}$ beam are shown as histograms.

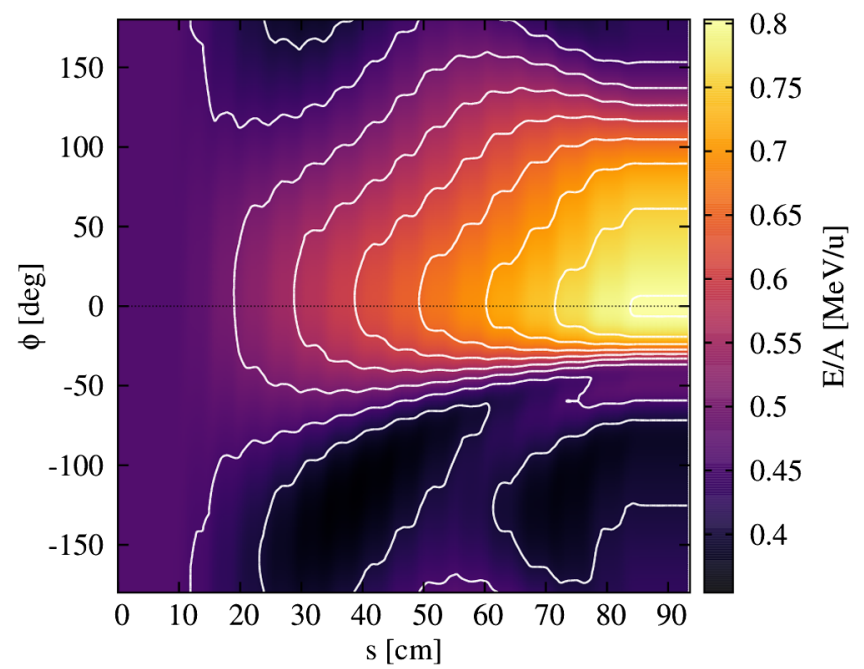

FIG. 4. TRANSOPTR computed reference particle $E / A$ for IH Tank-3, using the field shown in Fig. 3, center. The horizontal axis is the $s$-coordinate along the cavity's central optical axis, where the spatial component of the field is $\mathcal{E}_{\mathrm{n}}(s)$. Contours (white) delineate constant reference particle energy. The rf scaling parameter has been kept constant for each cavity phase $\phi$. The zero-degree phase causes monotonous energy increase along Tank-3. At the end of the cavity, the resulting $E / A$ vs $\phi$ response is characteristically nonsinusoidal, instead featuring a sharp dropoff in $E / A$ to phases negative of zero-degree. Phases above zero-degree cause a more gentle decrease in energy, until the output falls below the injection $E / A$ of $0.461 \mathrm{MeV} / u$. This characteristic profile is useful to establish a calibration between beam-based measurements and model computations, relating the field scaling in Eq. (8) and the rf amplitude parameter in the control system [38], given a computed field profile $\mathcal{E}_{\mathrm{n}}(s)$.

envelope-displaying and -optimizing web applications, which can poll the control system in real time. TRANSOPTR can use these setpoints to generate a live beam envelope, provided initial beam conditions. Finally, it is employed for on-line quadrupole scan tomography [4345], which uses beam profile monitors paired with quadrupoles to reconstruct the beam distribution in the machine. This provides us with a powerful tool for the computation and study of on-line tunes for the linac.

\section{B. Comparison with LORASR}

The envelope method's computational power is demonstrated using a full operational acceleration tune, which has been found using TRANSOPTR, constraining the optimizer to find a solution for optimum energy gain through each cavity and buncher, while also configuring the quadrupole triplets to obtain the transverse tune. Effective voltages and quadrupole gradients from the envelope code were then set directly in LORASR, producing $(x, y, z)$ envelopes shown in Fig. 5, with a transmission of $98 \%$ using 5000 particles. The starting $\boldsymbol{\sigma}$-matrix is listed in Table II. Beam envelopes 


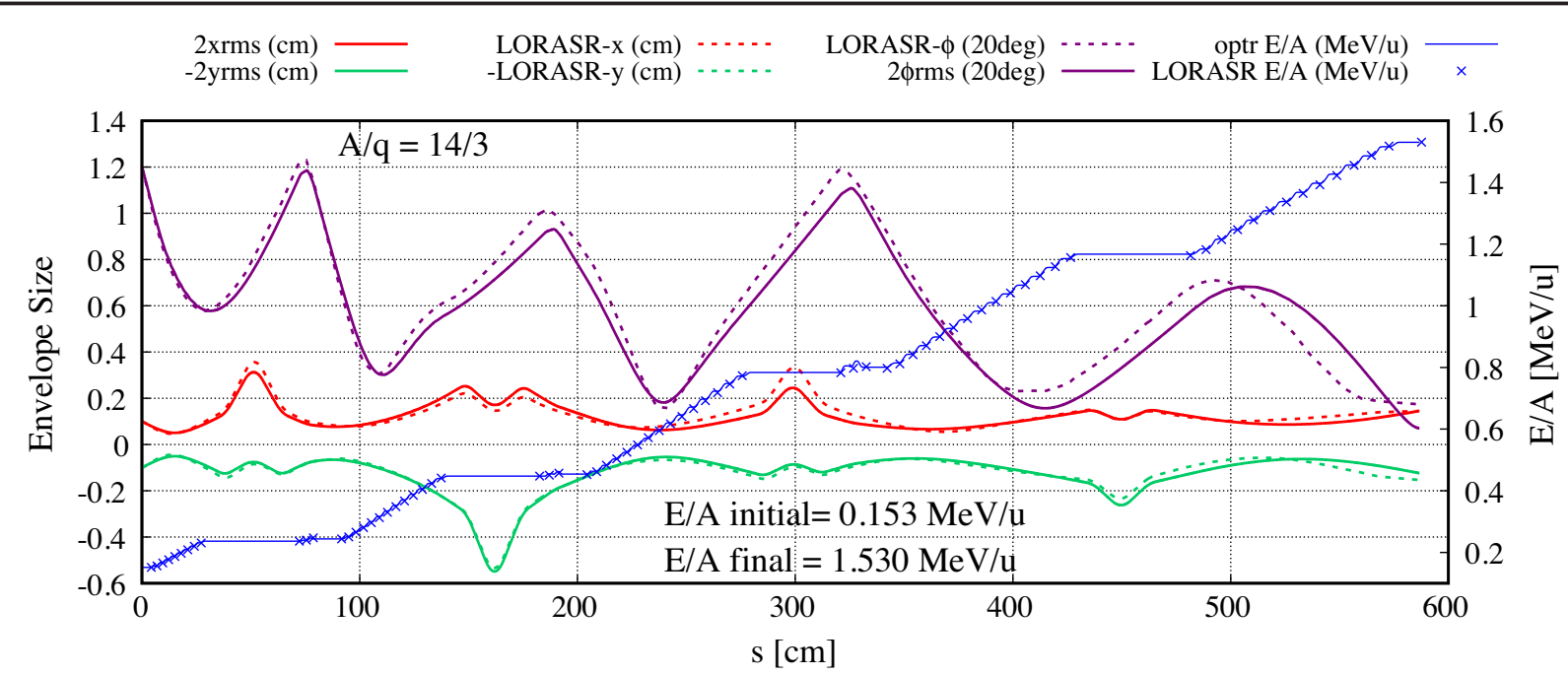

FIG. 5. Comparison of TRANSOPTR (solid, $2 \mathrm{rms}$ ) and LORASR (dashed, $96 \%$ containment) beam envelopes for ${ }^{14} \mathrm{~N}^{3+}$ through the ISAC-DTL, for an initial distribution listed in Table II. In this simulation, the ISAC-DTL is configured for an $E / A=1.530 \mathrm{MeV} / \mathrm{u}$ output, with tanks at negative synchronous phase. Longitudinal envelopes are presented in coordinate- 5 units of $20^{\circ}$ for plotting clarity, with $\phi=2 \pi z /(\beta \lambda)$.

in each dimension agree well while undergoing acceleration. This demonstrates the power of the envelope optimization method: TRANSOPTR's optimizer can return a full DTL tune in under roughly one minute. To showcase TRANSOPTR's behavior in presence of nonlinear forces and aberrations, the evolution of the longitudinal phase space in both codes is shown in Fig. 6. The injected coordinate 5,6 distribution suffers longitudinal nonlinearities from Tank-1 onward, resulting in broadening and deformation, which alters the spread of the distribution, while TRANSOPTR only tracks the linear forces. The computed tune features partial synchrotron oscillations through Tanks 4 and 5, resulting in a focus near the machine exit, at the cost of normalized

TABLE II. Input TRANSOPTR reference particle parameters and $\sigma$ matrix used for the comparative simulation shown in Figs. 5 and $6, a^{14} \mathrm{~N}^{3+}$ beam. Emittances are unnormalized.

\begin{tabular}{lcr}
\hline \hline Parameter & & Value \\
\hline$E_{i}$ & $(\mathrm{MeV})$ & 2.121 \\
$m_{0}$ & $\left(\mathrm{GeV} / \mathrm{c}^{2}\right)$ & 13.044 \\
$x_{i}$ & $(\mathrm{~cm})$ & 0.100 \\
$P_{x_{i}} / P$ & $(\mathrm{mrad})$ & 5.900 \\
$r_{12}$ & & -0.850 \\
$y_{i}$ & 0.100 \\
$P_{y_{i}} / P$ & $(\mathrm{~cm})$ & 5.900 \\
$r_{34}$ & $(\mathrm{mrad})$ & -0.850 \\
$\epsilon_{x, y}$ & & 0.311 \\
$z_{i}$ & $(\mathrm{~cm} \mathrm{mrad})$ & 0.170 \\
$P_{z_{i}} / P$ & $(\mathrm{~cm})$ & 6.133 \\
$r_{56}$ & $(\mathrm{mrad})$ & -0.848 \\
$\epsilon_{z}$ & & 0.554 \\
\hline \hline
\end{tabular}

longitudinal emittance growth of $40 \%$ in LORASR through the linac. This showcases the effects of second-order aberrations. While TRANSOPTR's optimizer can be constrained to minimize this [19], its envelope computation is first order, generated by the $\mathbf{F}$-matrix of Eq. (15). As is the case for the previously reported RFQ envelope implementation [21], the caveat remains that TRANSOPTR can faithfully simulate longitudinal bunch dynamics in the regime where linear forces are prevalent; the user must remain aware of this. As the model is intended for the computation of operational tunes on an existing machine, it is also true that these tunes are intended to operate in the linear regime where $z \ll \beta \lambda$, aiming to minimize higher order effects, as will be discussed. Both codes agree very well at optimum energy gain through the IH cavities under such conditions.

\section{Energy phase experiment}

KONUS achieves acceleration in the IH structures and reserves longitudinal focusing for the bunching sections. Second-order aberrations are minimized by running the IH cavities a few degrees off crest. At ISAC, during linac tuning, output energy is maximized while minimizing the energy spread [8]. As the field scaling and rf phase are changed, the $E / A$ response can cause significant transverse envelope size changes downstream, resulting in transmission loss. Since the quadrupoles in the structure are magnetic, the computation of the correct longitudinal energy profile along the structure is essential to find the transverse tune of the machine. Additionally, computation of the bunch $\left(z, P_{z}\right)$ extents, both of which depend on $\mathcal{E}_{\mathrm{n}}(s)$, are also essential to obtain the correct longitudinal tune. Both codes were used to perform cavity phase scans at fixed field scaling, in order to compare $E / A$ predictions. 

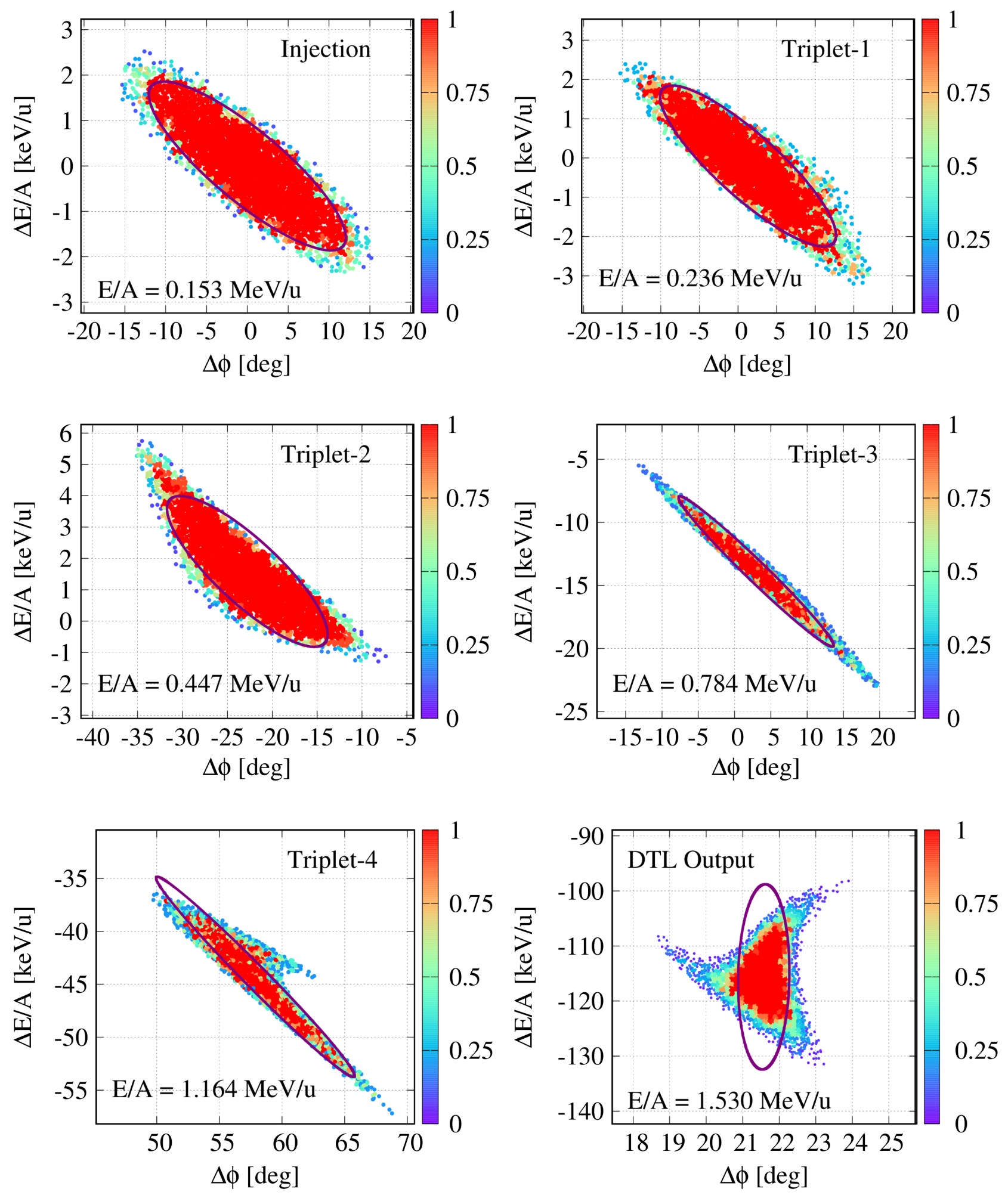

FIG. 6. Coordinate 5, 6 phase space distributions: LORASR normalized density for 5000 particles (points) and TRANSOPTR 4 rms containment ellipses (purple) at injection and through the ISAC-DTL, for the envelopes shown in Fig. 5, with input beam parameters shown in Table II. Both graph scales are with respect to the synchronous particle through each cavity, computed in LORASR. Normalized color density scale shown on the right of each plot. The intermediate distributions are shown at the exit of each triplet, with the energy per nucleon shown in each graph. As the bunch progresses through the linac, second-order components of the accelerating fields cause rf filamentation, which is represented in the multiparticle code. On the other hand, TRANSOPTR's ellipse arises only from the linear component of the forces, which nevertheless agrees with the core of the multiparticle beam simulation. The DTL output distribution consists of 10000 particles, highlighting the core of the distribution, where higher order effects are minimized. 

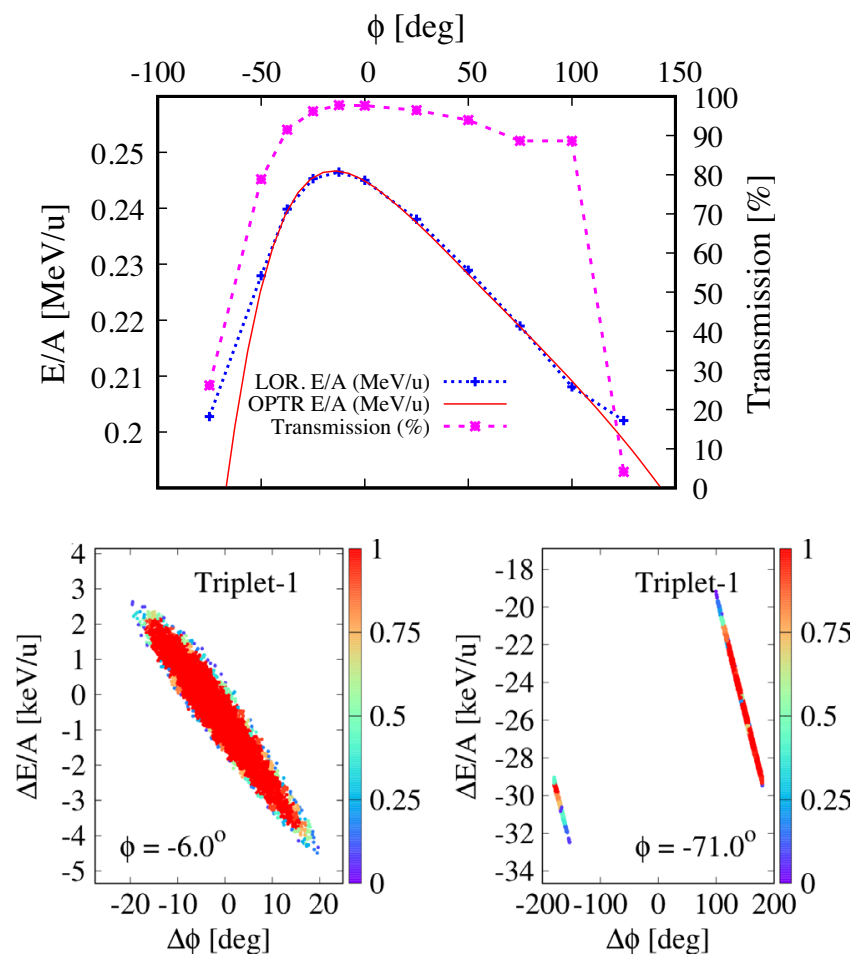

FIG. 7. Top: LORASR (blue) and TRANSOPTR (red) computed ISAC-DTL Tank-1 $E / A$ vs $\phi$ response and associated LORASR particle transmission (pink), which sharply drops at phases far from maximum acceleration. The near optimum distribution $\left(-6^{\circ}\right.$, bottom left) becomes severely broadened in time as the phase is detuned $\left(-71^{\circ}\right.$, bottom right), causing particle losses into adjacent buckets which also shifts the average energy.

LORASR's tracking of the average bunch center energy means transmission losses at phases sufficiently far away from zero-degree cause a systematic shift in output energy. Figure 7 shows the computed $E / A$ for DTL Tank- 1 in both codes, together with LORASR's beam transmission. As the rf phase is sufficiently detuned from zero-degree, nonlinearities in the rf waveform cause the $\left(z, P_{z}\right)$ distribution to exceed the bucket separatrix, causing extreme particles to lose synchronism with respect to the bunch distribution. Filamentation in the operational configuration of the ISACDTL emerges in the particle code, shown in Fig. 6, most prominently between triplets 2 to 4 , coinciding with the region of maximum $z$-envelope disagreement in Fig. 5. Some particles are also captured in adjacent rf buckets, as seen at the bottom right of Fig. 7. Overall this causes both a shift in the surviving average bunch energy and a drop in transmission. This occurs on either side of the zero-degree phase, though it is more severe to negative phases (early arrival). TRANSOPTR does not represent these effects, tracking only the reference particle and the linear forces around it, computed from the Hamiltonian [Eq. (14)].

DTL IH cavity phase scans were performed in both codes. The LORASR drift tube effective voltages were set to represent a flattened peak on axis electric field profile in each tank, while TRANSOPTR's fields have sagging profiles (Fig. 3). Additionally, using an ${ }^{16} \mathrm{O}^{4+}(A / q=4)$ beam from the offline ion source (Fig. 1, OLIS), the ISAC-DTL $E / A$ vs phase response was measured. The energy was recorded at the high-energy diagnostic station (Prague Magnet, Fig. 1), with a parallel wire detector which allows for a dispersive reading of both the centroid and spread of the energy distribution [46]. Beam measurements and model predictions are shown in Fig. 8. The data presents good overall agreement, replicating the characteristic phase response of each cavity at variable field scaling. Disagreements between models emerge in the form of depressions in the $E / A$ vs $\phi$ response for Tanks 1 to 4 , at positive phases and high scaling. On-line measurements agree better with TRANSOPTR's output $E / A$ than with LORASR's for all but Tank-3 [38]. This suggests the online field profiles of the remaining IH-cavities in the ISACDTL are well represented by the simulations of Fig. 3.

Both beam-based observations and a comparison with an established DTL code (Fig. 8) produce excellent agreement with TRANSOPTR envelopes and energy gain computations for IH cavities about maximum energy gain, validating its use for DTL tune computations. As noted, away from zerodegree acceleration, certain features of the measured $E / A$ response agree better with TRANSOPTR than with LORASR. The ISAC-DTL resonators were designed to maximize the flatness of the electric field [39], with this scenario implemented in the LORASR model. On the other hand, the OPERA2D fields used for the envelope computation feature constant tube voltages, which results in a sagging peak field intensity profile. This may reflect the true on-line condition of the fields within the resonators, now in service for two decades. Most notable are discrepancies with the measured $E / A$ of Tank-3 (Fig. 8), considering the good agreement between both models. This suggests a possible discrepancy between the assumed and actual on axis fields within the resonator, though further information is needed to better understand this. Consequently, this warrants future investigations of the cavity's performance and field. The comparisons presented in Fig. 8, together with Eq. (8) also hint at the possible future use of such scans together with TRANSOPTR in an attempt to derive information on the status of the on axis electric field, with the beam's $E / A$ response as a probe. Under this scheme, different simulated cavity electric fields representing a variety of anomalous conditions, for example drift tube voltage deviations on the order of $10 \%$ from design (deteriorated rf contact between tubes and ridges, change in surface properties, etc.), would be used in TRANSOPTR, to find the best possible match with on-line datasets. Despite this, the energy-phase profiles agree reasonably well around maximum acceleration for Tank-3 at low to intermediate scaling. A CST-Mws model of the DTL cavities is being developed to study the systematic effects of changes to the resonators upon both $\mathcal{E}(s)$ and the machine optics. 

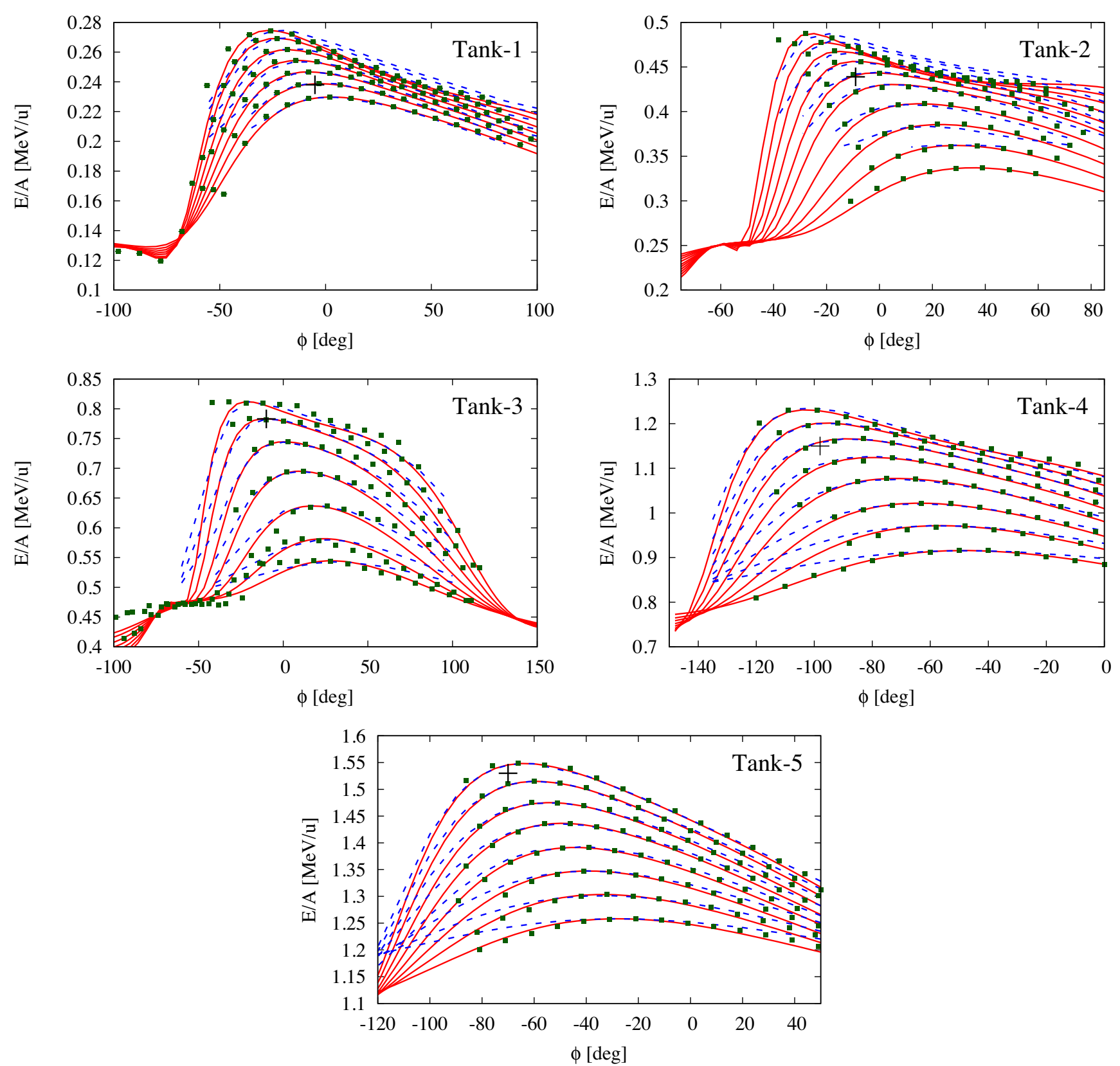

FIG. 8. TRANSOPTR (red) and LORASR (blue) simulations of ISAC-DTL IH-Tank output $E / A$ at variable rf phase but fixed on axis electric field scaling $V_{0}$. ISAC-DTL measured ${ }^{16} \mathrm{O}^{4+} E / A$ response superimposed in green. The LORASR model assumes perfect field flatness, while the TRANSOPTR model features a sagging in the maximum peak amplitude (Fig. 3). The setpoint of each tank at maximum operational acceleration (Table I) is shown as a black cross on each figure. Note the $x$ axis phases are absolute, meaning optimum acceleration is not necessarily at $0^{\circ}$.

\section{AUTOFOCUS}

Since it employs an infinitesimal transfer matrix approach, the computation returns the global transfer matrix, connecting each point on the reference orbit to the start of the simulation, including inside accelerating fields. The internal optimizers can be used to impose user specified constraints upon either the beam or transfer matrices in the linac. For instance, optimum acceleration is obtained by minimizing rf defocusing through the cavity, at any desired output $E / A$. The IH cavity constraint can be understood by considering the infinitesimal transfer matrix of Eq. (15) and the envelope equation (3): given any field scaling, optimum acceleration occurs at a phase which maximizes the energy gain, while also minimizing the accumulated transverse and longitudinal transfer matrix 


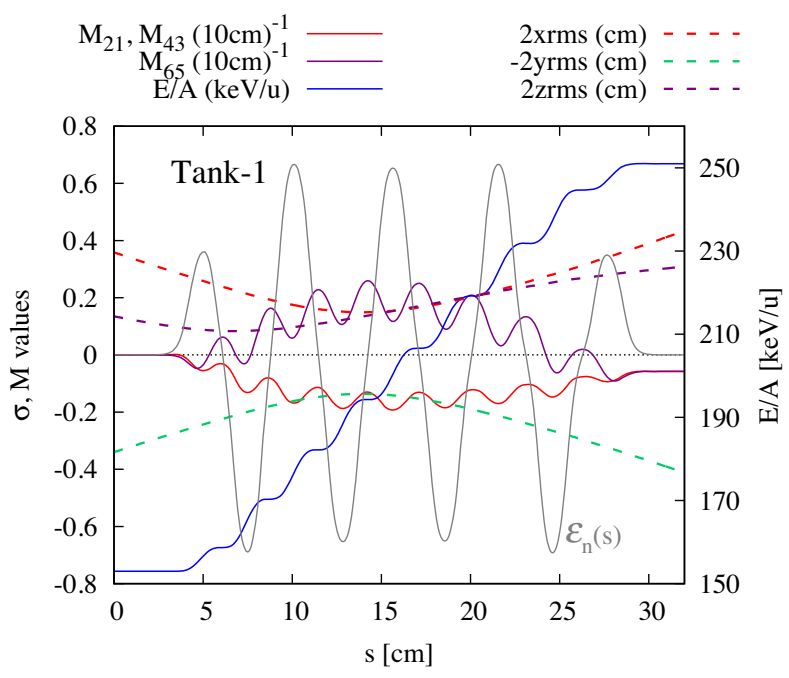

FIG. 9. TRAnSOPTR computed elements $\mathbf{M}_{21}, \mathbf{M}_{43}$, and $\mathbf{M}_{65}$ (solid lines) across DTL Tank-1. Optimum acceleration to $E / A=$ $0.251 \mathrm{MeV} / \mathrm{u}$ is shown, where the energy gain (blue, right $y$ axis) is monotonous, minimizing longitudinal restoring forces, which in turn reduces transverse $\mathrm{rf}$ focal effects. The plot also shows the $(x, y, z) 2$ rms envelopes, each as dotted lines. The scaled value of Tank-1 $\mathcal{E}_{\mathbf{n}}(s)$ is shown in grey.

effects of $\mathcal{A}(s)$ and $\mathcal{B}(s)$ from Eq. (16). Figure 9 shows the transfer matrix element $\mathbf{M}_{21}\left(\mathbf{M}_{43}=\mathbf{M}_{21}\right)$ and $\mathbf{M}_{65}$ through Tank-1, with the reference particle $E / A$ shown on the right hand $y$ axis. Both $\mathbf{M}_{21}$ and $\mathbf{M}_{65}$ have been minimized by TRANSOPTR's simulated annealing routine, while maximizing energy gain through the tank, for a desired $E / A=0.251 \mathrm{MeV} / \mathrm{u}$. The optimization was completed in 23 iterations, varying the field scaling and phase and solving the equations of motion. The optimal solution keeps the longitudinal distribution within the region of linear forces, reducing any higher order effects and transverse $\mathrm{rf}$ defocusing. Any of the IH cavities can be optimized by using TRANSOPTR to find $\left(\phi, V_{0}\right)$ pairs satisfying this simple constraint, at any desired output energy: $\mathbf{M}_{21}+$ $\mathbf{M}_{43}+\mathbf{M}_{65} \approx 0$. It is not exactly zero due to the necessity for a small amount of longitudinal bunching, which induces defocusing $\mathrm{rf}$ forces.

Variable energy KONUS linacs by their nature require frequent tune recomputations. In order to compute these for the ISAC-DTL following energy changes, TRANSOPTR is called upon to sequentially optimize groups of adjacent devices [47] (quadrupoles, IH cavities, bunchers, etc.), to obtain the desired envelope. This is advantageous since it minimizes the burden on the optimizer, which is provided with a smaller subset of variables at each call, increasing the overall speed of the operation. Terminating the simulation after the last fit constraint avoids wasting resources downstream. For the ISAC-DTL, the TRANSOPTR tuning sequences necessary to establish the transverse and longitudinal optimum in the linac are listed in Table III and shown in Fig. 10. This allows for the use of TRANSOPTR at
TABLE III. Tune sequences for the ISAC-DTL autofocus, specifying the devices that are to be optimized by TRANSOPTR. The sequences are shown in Fig. 10 and allow for the rapid computation of the ISAC-DTL optics at any $E / A$.

\begin{tabular}{ccc}
\hline \hline Cavity & $E / A[\mathrm{MeV} / \mathrm{u}]$ & Sequence (Fig. 10) \\
\hline Tank-1 & 0.254 & $1 a, 1 b, 1 c, 1 d, 1 e$ \\
Tank-2 & 0.461 & $1 a, 1 b, 2 a, 2 b, 2 c, 2 d$ \\
Tank-3 & 0.803 & $1 a, 1 b, 2 a, 2 b, 3 a, 3 b, 3 c$ \\
Tank-4 & 1.149 & $1 a, 1 b, 2 a, 2 b, 3 a, 3 b, 4 a, 4 b$ \\
Tank-5 & 1.530 & $1 a, 1 b, 2 a, 2 b, 3 a, 3 b, 4 a, 4 b, 5 a$ \\
\hline \hline
\end{tabular}

the heart of a DTL autofocusing capability, which can compute the machine optics at any $A / q$ and any output energy. The technique of sequential tune optimization in TRANSOPTR is general and not limited to drift tube structures. The low-energy segments of the linac can also be retuned in this manner [48]. Additionally, envelopes of much larger systems including a DTL can now be computed. Since every device is treated through the same

(a)

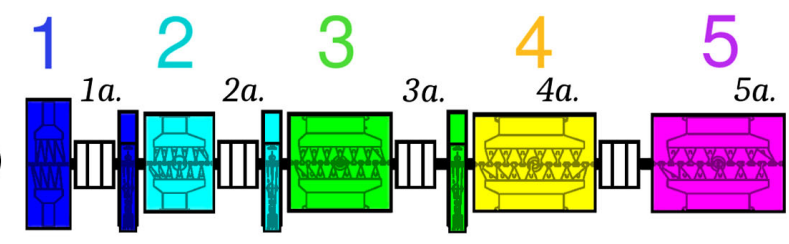

(b)

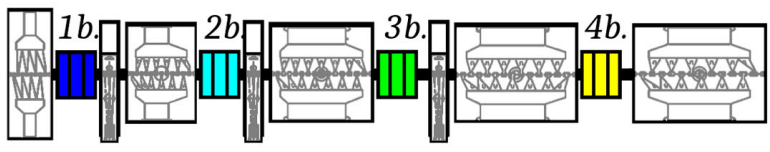

(c)

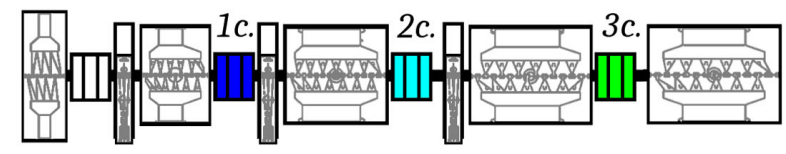

(d)

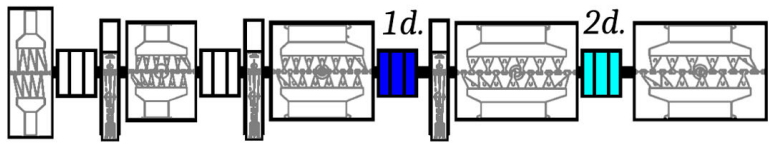

(d)

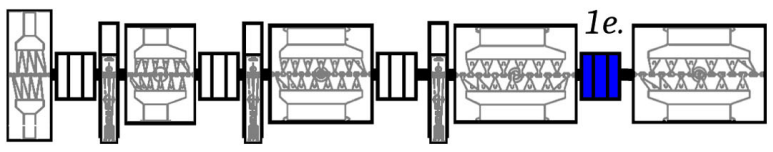

FIG. 10. Graphical representation of tuning sequences from Table III for various ISAC-DTL configurations, corresponding to Tank 1 to $5 E / A$ cases (Table I). Colors represent optimization at a given energy, e.g., dark blue is up to $0.254 \mathrm{MeV} / \mathrm{u}$. The optimizer is called upon in the sequence $a$ to $e, 1$ to 5. Step $a$ corresponds to longitudinal tank configuration, while steps $\{b, c, d, e\}$ accomplish DTL triplet tuning, in each case using TRANSOPTR's optimizer. Tanks 1 to 3 are optimized with their bunchers. 

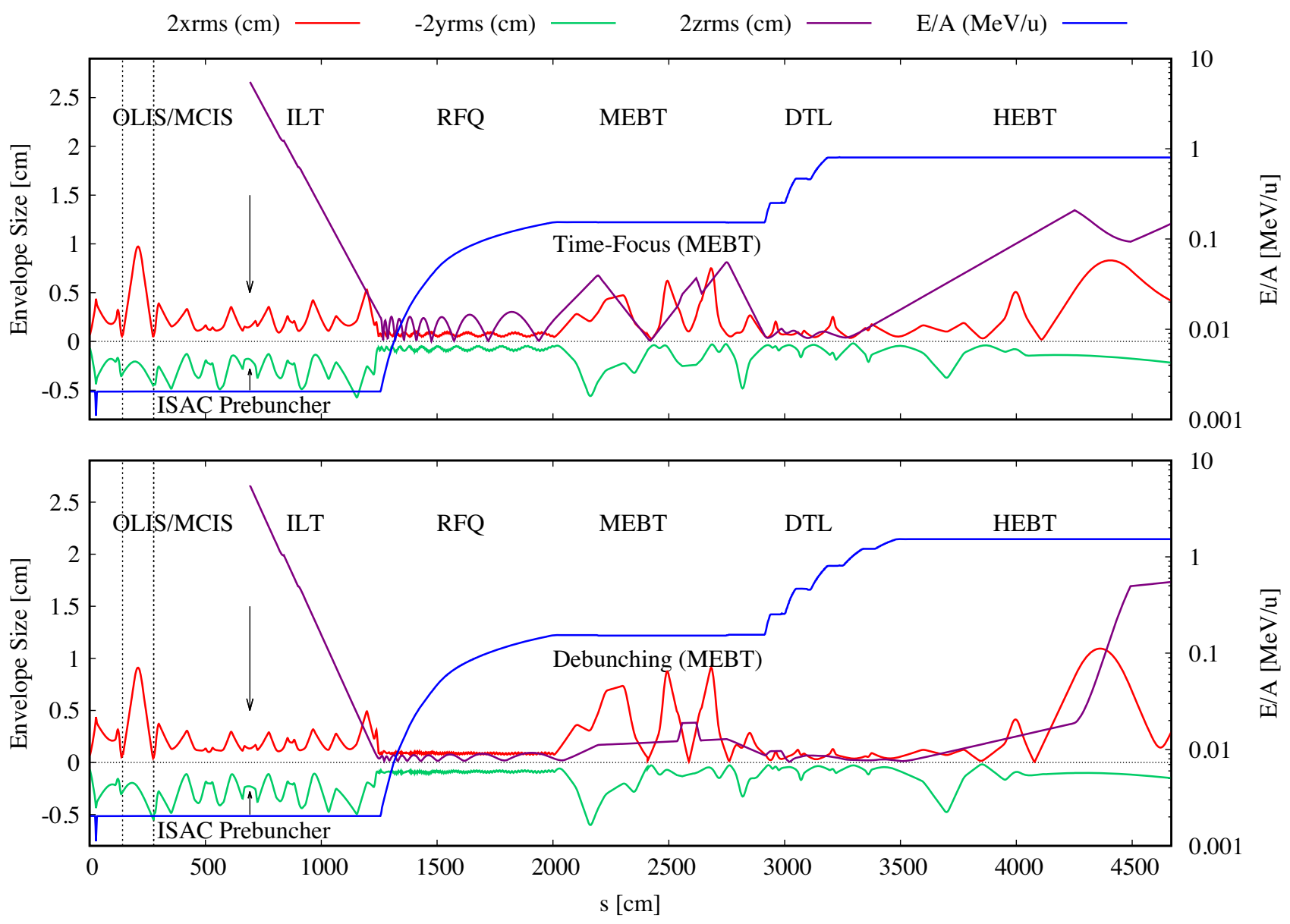

FIG. 11. TRANSOPTR computations of $2 \mathrm{rms}$ envelopes and reference particle energy for a ${ }^{20} \mathrm{Ne}^{4+}$ beam in the ISAC-I linac, from OLIS extraction at $E / A=2.04 \mathrm{keV} / \mathrm{u}$ up to the Prague Magnet station (Fig. 1). The dip in $E / A$ near $s=0$ is from a decelerating Einzel lens [53]. The OLIS slits are shown as vertical dashed lines. The code is run in 6D bunched mode, with initial longitudinal beam matrix values chosen to mimic the acceptance of the prebuncher, which is represented as a single discrete $11 \mathrm{MHz} \mathrm{rf}$ kick (arrows), though OLIS beams are continuous. The starting energy spread is equal to the prebuncher's three harmonic waveform deviation from a perfect sawtooth. The top shows the ISAC-DTL configured for acceleration up to $0.803 \mathrm{MeV} / \mathrm{u}$. The DTL triplets and HEBT optics have been reoptimized, along with Tanks 4 and 5, to retune the linac to $1.53 \mathrm{MeV} / \mathrm{u}$ acceleration in the bottom figure. The longitudinal mismatch into the RFQ is corrected by optimizing the prebuncher and low-energy optics. Additionally, the MEBT time focus (top) has been retuned to debunch the beam longitudinally (bottom), requiring a change in MEBT \& DTL rf settings. The high-energy analyzing magnet (Fig. 1) is located between $s=4200$ and $4600 \mathrm{~cm}$, upstream of the parallel wire detector.

formalism, it is straightforward to simulate and compute corrections for intercavity effects, mismatches or time of flight effects in global linac tunes. Figure 11 shows end-toend beam envelopes in the ISAC-I linac from the OLIS multicharge ion source (MCIS) up to the high-energy analyzing magnet. The $E / A$ changes from $2.04 \mathrm{keV} / u$ to Tank-3 (Fig. 11, top) and Tank-5 (same fig., bottom) acceleration values (Table I), for a ${ }^{20} \mathrm{Ne}^{4+}$ beam. In both computations, the beam energy changes by nearly three orders of magnitude and includes 12 separate rf cavities and over 70 quadrupoles. The code's RK engine used $10^{5}$ steps to solve the envelope Eq. (3), with $\epsilon$ set to $10^{-4}$. A synchrobetatron oscillation arising from a mismatch into the RFQ [21] can be seen at the top of Fig. 11. The match is improved by optimizing the low-energy section (OLIS \& ILT) quadrupoles and the prebuncher in the bottom of the figure. The time-focus in MEBT is changed to cause debunching, requiring reoptimization of downstream $\mathrm{rf}$ cavities, along with MEBT quadrupoles. The DTL and HEBT quadrupoles have also been reoptimized. The code includes a slit or aperture subroutine [49], which cuts the beam distribution according to a user-specified $(x, y)$ aperture, marked as vertical dotted lines in Fig. 11, enabling studies of beam loss patterns [50]. An approximation for anticipated transmission is obtained by measuring the ratio of initial and final transverse emittances. Input beam matrices for different sections can be obtained through various on-line diagnostics, feeding the TRANSOPTR model 
with real-time data for simulations of the anticipated state of the machine during operation, enabling comparison of expected envelope size with the rms size of measured beam distributions. Arbitrary charge states can be handled, allowing for investigations of the anticipated behavior of a diversity of $A / q$ envelopes in the same linac tune. This enables studies of multiple $A / q$ beams $[51,52]$ in a DTL structure.

\section{CONCLUSION}

Beam-envelope modeling of variable energy KONUS DTLs, now possible in TRANSOPTR, opens up a diverse array of possibilities for the analysis and exploitation of such designs. The method does not rely upon "transit time factors," but rather uses the on axis field to calculate energy gain, as was validated at TRIUMF-ISAC and with the code LORASR. A novel envelope autofocusing capability, using TRANSOPTR's optimizers to rapidly recompute optimal machine envelopes at any energy, was presented. This approach can be applied to any DTL, provided knowledge of $\mathcal{E}(s)$. Full end-to-end simulations and optimizations of the linear optics of larger accelerator chains, including DTLs, are possible thanks to the lower computational burden of the envelope method. These include the desired output energy, the setting of waists or establishment of achromaticity in sections. A beam-based calibration enables use of TRANSOPTR's internal optimizers for the rapid computation of on-line DTL cavity amplitude settings for acceleration. Using on-line beam diagnostic measurements to provide TRANSOPTR with initial values allows for realtime machine simulation and optimization. The versatility of TRANSOPTR and the computational efficiency of this method are also ideal for the training of a neural network, supporting machine learning investigations for accelerator tuning, which can now include DTL envelopes.

\section{ACKNOWLEDGMENTS}

Thanks to R. Laxdal, B. Waraich, M. Marchetto, R. Leewe, Z. Ang, T. Au, T. Emmens, V. Toma, and all operators. C. Barquest is thanked for assistance with code development. TRIUMF is funded under a contribution agreement with NRC (National Research Council Canada.) The TRIUMF campus is located on the traditional, ancestral, and unceded territory of the Musqueam people.

[1] R. Laxdal, Acceleration of radioactive ions, Nucl. Instrum. Methods Phys. Res., Sect. A 204, 400 (2003).

[2] O. Kester et al., Accelerated radioactive beams from REXISOLDE, Nucl. Instrum. Methods Phys. Res., Sect. A 204, 20 (2003).
[3] U. Ratzinger, K. Kaspar, E. Malwitz, S. Minaev, and R. Tiede, The GSI $36 \mathrm{MHz}$ high-current IH-type RFQ and HIIF-relevant extensions, Nucl. Instrum. Methods Phys. Res., Sect. A 415, 281 (1998).

[4] P. N. Ostroumov, T. Maruta, S. Cogan, K. Fukushima, S. H. Kim, S. Lidia, F. Marti, A. S. Plastun, J. Wei, T. Yoshimoto, T. Zhang, and Q. Zhao, Beam commissioning in the first superconducting segment of the facility for rare isotope beams, Phys. Rev. Accel. Beams 22, 080101 (2019).

[5] R. Laxdal, P. Bricault, T. Reis, and D. Gorelov, A separated function drift-tube linac for the ISAC project at TRIUMF, in Proceedings of the 1997 Particle Accelerator Conference (Cat. No. 97CH36167), Vol. 1 (IEEE, Vancouver, BC, 1997), pp. 1194-1196.

[6] U. Ratzinger, H. Hähnel, R. Tiede, J. Kaiser, and A. Almomani, Combined zero degree structure beam dynamics and applications, Phys. Rev. Accel. Beams 22, 114801 (2019).

[7] K. Fong, M. Laverty, and S. Fang, RF control systems for the TRIUMF ISAC RF structures, in PACS2001 Proceedings of the 2001 Particle Accelerator Conference (Cat. No. 01CH37268)(IEEE, Chicago, IL, 2001), pp. 948-950, Vol. 2 .

[8] M. Marchetto, J. Berring, and R. E. Laxdal, Upgrade of the ISAC DTL tuning procedure at TRIUMF, Conf. Proc. C 0806233, THPP031 (2008).

[9] M. Marchetto, R. E. Laxdal, and F. Yan, Decelerating heavy ion beams using the ISAC DTL, in Proceedings of 11th International Conference on Heavy Ion Accelerator Technology (HIAT'09) (JACoW Publishing, Geneva, Switzerland, 2009), pp. 261-265.

[10] O. Shelbaya, TRANSOPTR implementation of the MEBT beamline, TRIUMF, Vancouver, Canada, Technical Report No. TRI-BN-19-02, 2019.

[11] M. Pasini, R. Laxdal, and P. Ostroumov, Beam dynamics studies on the ISAC-II post-accelerator at TRIUMF, in Proceedings of EPAC 2002 Conference, Paris, France (JACOW Publishing, Geneva, Switzerland, 2002), pp. 933-935.

[12] D. Gorelov, P. Ostroumov, and R. Laxdal, Use of the LANA code for the design of a heavy ion linac, in Proceedings of the 1997 Particle Accelerator Conference (Cat. No. 97CH36167), Vol. 2 (IEEE, Vancouver, BC, 1997), pp. 2621-2623.

[13] R. Tiede, G. Clemente, H. Podlech, U. Ratzinger, A. C. Sauer, and S. Minaev, LORASR code development, Conf. Proc. C 060626, 2194 (2006).

[14] R. Tiede et al., Improvements of the LORASR code and their impact on current beam dynamics designs, Technical Report No. TUPP063, in Proceedings of LINAC 2014, Geneva, Switzerland (JACOW, Geneva, Switzerland, 2014).

[15] O. Shelbaya and O. Kester, Toward an end-to-end model for ISAC-I accelerators, J. Phys. Conf. Ser. 1067, 062028 (2018).

[16] E. Heighway and R. Hutcheon, TRANSOPTR - A second order beam transport design code with optimization and constraints, Nucl. Instrum. Methods Phys. Res., Sect. A 187, 89 (1981). 
[17] E. Heighway and M. De Jong, TRANSOPTR-A beam transport design code with space charge, automatic internal optimization and general constraints, Technical Report No. AECL-6975 (rev.A), Atomic Energy of Canada Limited, Chalk River, Ontario, 1984.

[18] TRIUMF TRANSOPTR open source git repository, https:// gitlab.triumf.ca/beamphys/transoptr.

[19] R. Baartman, TRANSOPTR: Changes since 1984, TRIUMF, Vancouver, Canada, Technical Report No. TRI-BN16-06, 2016.

[20] R. Baartman, Bunch dynamics through accelerator column, in Particle accelerator, in Proceedings of 2 nd International Conference, IPAC 2011, San Sebastian, Spain, 2011 (2011), Vol. C110904, pp. 649-651.

[21] O. Shelbaya, R. Baartman, and O. Kester, Fast radio frequency quadrupole envelope computation for model based beam tuning, Phys. Rev. Accel. Beams 22, 114602 (2019).

[22] V. Fields, Opera-2d user guide (Vector Fields Limited, England, 1999).

[23] M. De Jong and E. Heighway, A first order space charge option for transoptr, IEEE Trans. Nucl. Sci. 30, 2666 (1983).

[24] R. Baartman, Bunched beam space charge in TRANSOPTR, TRIUMF, Vancouver, Canada, Technical Report No. TRI-BN-21-04, 2021.

[25] S. Penner, Calculations of properties of magnetic deflection systems, Rev. Sci. Instrum. 32, 150 (1961).

[26] D. C. Carey, Karl Brown's Role in TRANSPORT, Phys. Today 56, 14 (2003).

[27] K. L. Brown, A first and second order matrix theory for the design of beam transport systems and charged particle spectrometers, Adv. Part. Phys. 1, 71 (1968).

[28] H. Wollnik, J. Brezina, and C. Geisse, GIOS- a program for the design of ion optical system, ii, Physikalisches Institut, Universität Giessen D6300 Giessen, 1987.

[29] F. J. Sacherer, RMS envelope equations with space charge, IEEE Trans. Nucl. Sci. 18, 1105 (1971).

[30] P. M. Lapostolle, Quelques propriétés essentielles des effets de la charge d'espace dans les faisceaux continus, Technical Report No. CERN-ISR-DI-70-36, ISR-DI-7036, CERN, Geneva, 1970.

[31] I. Kapchinskij and V. Vladimirskij, Limitations of proton beam current in a strong focusing linear accelerator associated with the beam space charge, in Proceedings of 2nd International Conference on High-Energy Accelerators and Instrumentation, HEACC 1959: CERN, Geneva, Switzerland, 1959 (CERN, Geneva, 1959), pp. 274-287.

[32] R. Baartman, Fast envelope tracking for space charge dominated injectors, in Proceedings of LINAC 2016 Conference, East Lansing, MI (Michigan State University, Geneva, Switzerland, 2016), pp. 1017-21.

[33] R. Baartman, Runge-Kutta customization for TRANSOPTR, TRIUMF, Vancouver, Canada, Technical Report No. TRI-BN-18-09, 2018.

[34] K. L. Brown, D. C. Carey, F. C. Iselin, and F. Rothacker, TRANSPORT: A computer program for designing charged-particle beam-transport systems, CERN Yellow
Report No. 80-04, CERN, Geneva, 1980, SLAC-91 and FERMILAB NAL-91.

[35] R. Poirier, P. Bricault, K. Fong, A. Mitra, H. Uzat, and Y. V. Bylinsky, RF systems of the TRIUMF ISAC facility, in Proceedings of the 1999 Particle Accelerator Conference (Cat. No. 99CH36366) (IEEE, New York, NY, 1999), Vol. 1, pp. 450-452.

[36] R. Laxdal et al., A 3-gap booster cavity to match ion source potential to RFQ acceptance, in Proceedings of 29th Linear Accelerator Conference (LINAC'18), Beijing, China (2018), pp. 196-199.

[37] R. Laxdal, Charge distribution and beam loss after MEBT foil, TRIUMF, Vancouver, Canada, Technical Report No. TRI-DN-99-27, 1999.

[38] O. Shelbaya, The TRANSOPTR model of the ISAC drift tube linear accelerator-Part I: Longitudinal verification, TRIUMF, Vancouver, Canada, Technical Report No. TRIBN-20-08, 2020.

[39] P. Bricault et al., Status report on the construction of the ISAC drift tube LINAC, eConf C000821 (2000) MOD03.

[40] A. Mitra et al., RF test and commissioning of the radio frequency structures of the TRIUMF ISAC I facility, in Proceedings of LINAC 2002 (Citeseer, Pohang, Republic of Korea, 2002), p. 106.

[41] R. Laxdal, Concept design for quadrupole triplets for the ISAC separated function drift tube linac, TRIUMF, Vancouver, Canada, Technical Report No. TRI-DN-9905, 1999.

[42] A. Vasyuchenko, A. Feschenko, A. Kvasha, A. Menshov, V. Paramonov, Y. Bylinsky, G. Dutto, R. Laxdal, A. Mitra, and R. Poirier, Development, Fabrication and test of triple gap split-ring bunchers for the TRIUMF ISAC facility, Conf. Proc. C 0106181, 978 (2001).

[43] Y. Rao, Maximum entropy tomography validation, TRIUMF, Vancouver, Canada, Technical Report No. TRI-BN18-16, 2018.

[44] S. Saminathan, F. Ames, R. Baartman, M. Marchetto, O. Lailey, and A. Mahon, Tomography reconstruction of beams extracted from an ion source, Rev. Sci. Instrum. 90, 123302 (2019).

[45] O. Shelbaya et al., On-line retuning of ISAC linac beam with quadrupole scan tomography, in Proceedings of 11th International Particle Accelerator Conference (IPAC'21), Campinas, SP, Brazil, 2021 (JACOW Publishing, Geneva, Switzerland, 2021).

[46] M. Marchetto and R. Laxdal, High energy beam lines, in ISAC and ARIEL: The TRIUMF Radioactive Beam Facilities and the Scientific Program (Springer, New York, 2013), pp. 99-109.

[47] O. Shelbaya, Sequential tune optimization with TRANSOPTR, TRIUMF, Vancouver, Canada, Technical Report No. TRI-BN-20-14, 2020.

[48] O. Shelbaya, Status of model coupled accelerator tuning at ISAC-I, TRIUMF, Vancouver, Canada, Technical Report No. TRI-BN-21-07, 2021.

[49] R. Baartman, SLIT routine for TRANSOPTR, TRIUMF, Vancouver, Canada, Technical Report No. TRI-BN-19-21, 2019. 
[50] O. Shelbaya, Anomalous operational OLIS tunes, TRIUMF, Vancouver, Canada, Technical Report No. TRI-BN19-20, 2019.

[51] P. N. Ostroumov, R. C. Pardo, G. P. Zinkann, K. W. Shepard, and J. A. Nolen, Simultaneous Acceleration of Multiply Charged Ions through a Superconducting Linac, Phys. Rev. Lett. 86, 2798 (2001).
[52] Z. He, Y. Zhang, J. Wei, Z. Liu, and R. Talman, Linear envelope model for multicharge state linac, Phys. Rev. ST Accel. Beams 17, 034001 (2014).

[53] O. Shelbaya, OLIS to RFQ beam transport and acceleration in TRANSOPTR, TRIUMF, Vancouver, Canada, Technical Report No. TRI-BN-20-13, 2020. 